\title{
Challenges and Solutions in Customer Process Based on Collaborative Engineering
}

\author{
Sergej Bondar ${ }^{1}$, Leo Potjewijd ${ }^{2}$, and Josip Stjepandic ${ }^{1}$ \\ ${ }^{1}$ PROSTEP AG, Dolivostraße 11, 64293 Darmstadt, Germany \\ \{sergej . bondar, josip.stjepandic\} @opendesc.com \\ ${ }^{2}$ SKF AB, Kelvinbaan 16, 3430 DT Nieuwegein, Netherlands \\ leo.potjewijd@skf.com
}

\begin{abstract}
Due to the higher-level business trends, such as increasing globalization and outsourcing, the collaborative engineering gets more and more important. This increases the challenge for the suppliers to accomplish their customer processes optimally with minimal effort. Efficient data communication in all phases of the product development process is a prerequisite for lean and flexible collaboration processes.

In this paper, specific concepts and solutions are described, as this problem can be solved from the perspective of a tier-1 supplier by using advanced IT technology based on contemporary standards. As a case study, the example of the company SKF is shown, one of the major bearing manufacturers for the automotive sector that develops on 14 locations worldwide and communicates with over 50 OEMs (original equipment manufacturer) synchronously. The recently implemented scalable solution fosters the customer process by ensuring the data communication in the right format, quality and time and achieving significant cost savings and error reduction.
\end{abstract}

Keywords: Collaborative engineering, distributed development, CAD data exchange, CAD data translation.

\section{Introduction}

Nowadays modern manufacturing industries like automotive are acting in the dynamic global marketplace that demands instant responses to customer requirements, short time-to-market and high flexibility in production. In the past decades the manufacturing industries were even more shifting to a distributed environment like an extended enterprise with increasing agility. We set the customer's satisfaction on the first place in order to get the assignment against many competitors. This has caused the mass customization at high level and even more complex development, manufacturing as well as logistics processes along the manufacturing supply chain. Thus the upcoming outsourcing has derived a multi-tier supply network structure involving numerous enterprises around the globe. The product development as well takes place in global development partnerships. OEMs accomplish the development of new products at many locations in several countries across the world [1]. Furthermore a variable 
number of external service providers and suppliers take part in individual projects. The most relationships in this supply network are temporary, exceed by the end of project and today's project partner can quite become tomorrow's harshest competitor (Fig. 1).

The suppliers were involved in the product development processes as early as possible, because they mostly possess a greater depth of domain expertise, which is necessary for best product development. The OEM-supplier relationship is characterized by a sequential interaction whereby the OEM gives clear product and production requirements to the supplier and the supplier delivers the product or service to the OEM. Supplier integration is a crucial method for incorporating a supplier's innovativeness in the product development process and reduce the costs and risk [2]. It also creates synergy through mutually interacting deliverables and decisions between OEM and suppliers. Both sides take advantage of each other's capability to develop the product as well as to obtain feedback from the other party to improve the product development. Due to the complex development cycle, the OEM took the lead and has begun to adopt supplier integration into its product development process. To respond to this trend, the collaboration and partnership management between the OEM and suppliers need to be continuously improved to reduce costs and time. Regarding the depth of collaboration, the integration of suppliers into the OEM process chain can be defined in many ways, depending on corresponding work package and type of collaboration [3]. To enable the success of supplier integration, this work describes how to shape the collaboration between the OEM and its suppliers, through determining an appropriate supplier integration method and using the proper tools.

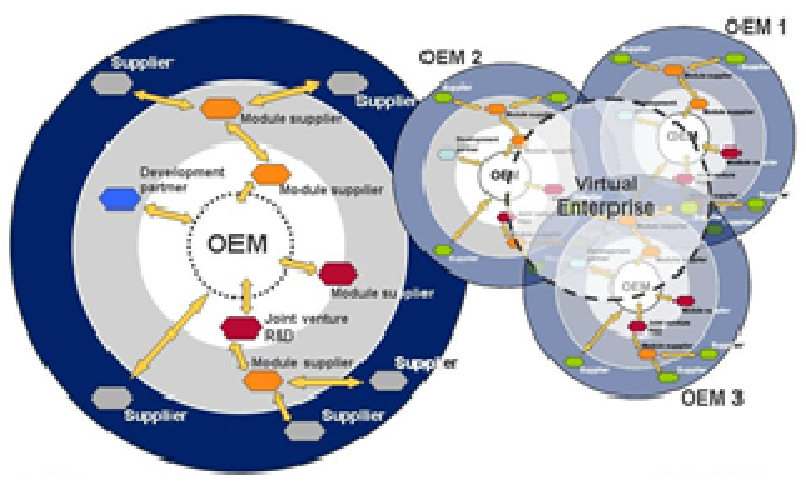

Fig. 1. Virtual enterprise in automotive industry

In the context of concurrent and collaborative engineering, the validity and consistency of product information become important. However, it is difficult for the current computer-aided systems to check the information validity and consistency because the engineers' intent is not fully represented in a consistent product model. Due to the different approaches and IT systems in the automotive OEM industry, a unified solution is not possible at this time. In particular, automotive suppliers that develop system components for a number of different OEMs or tier-1 suppliers, face 
the challenge of ensuring that they make the CAD data available in the format required by their customers and with a high level of reliability and, if data translation is involved, that they take the system configuration of the respective customer into consideration. Special requirements like the flexibility for further changes in business relationships and the intellectual property protection are also taken into account.

\section{Cooperation Models}

As the outsourcing became standard business approach in the manufacturing industries, many industry associations like the German automotive manufacturers association (VDA) accomplished the basic development work to define and classify the typical collaboration models and corresponding processes [4] (Figure 2). There are 6 supplier types defined according to the criteria: production technical integration, process integration, functional integration, and geometrical (spatial) integration of the whole product (car). Beside of the prime contractor who can be defined as a clone of the OEM without the product management, sales and marketing function, all other types of suppliers (system supplier, module supplier, component supplier, part supplier, and engineering service provider) maintain a high level of independency in their corresponding processes. Taking the fact into account that a supplier supplies many customers, who have their own, various processes and infrastructures, there yields a strong need for a comprehensive integration approach based primarily on standards and serving the relationships to all the customers.

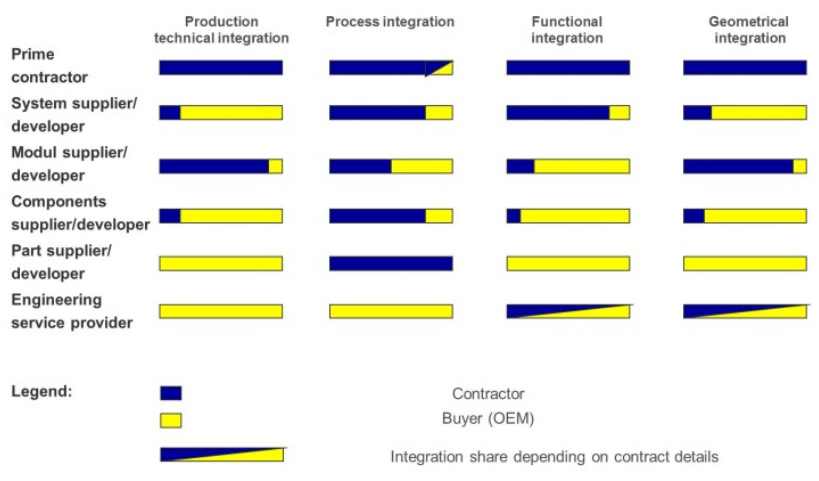

Fig. 2. Virtual enterprise in automotive industry

The responsibility of suppliers to provide the suitable product documentation in each phase of product emerging process is self-evident for an OEM. Thus the supplier is forced to take the corresponding measures to adapt his data to OEM's requirements.

\section{Case SKF}

The SKF Group is one of the leading manufacturers of precision bearings, seals, mechatronic components and lubrication systems. It also offers customers a wide range 
of services including maintenance services and condition monitoring. Founded in 1907 in Sweden, the company is a global player with 110 manufacturing sites distributed around the world and represented in over 130 countries. In the 2012 business year, the Group, which has over 46,000 employees, generated sales of 64.6 billion Swedish krona or the equivalent of 7.1 billion euros.

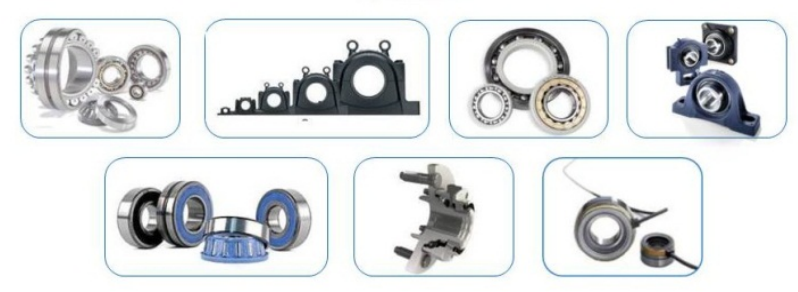

Fig. 3. Product platform "Bearing and units" at SKF

SKF is organized into 2 Markets servicing more than 40 customer segments. While the Industrial Market takes care of industrial original equipment manufacturers (OEMs) in the energy, railway vehicle, machine tool, medical, food and beverage and other industries, as well as the respective aftermarkets, the Automotive Market supplies manufacturers of passenger cars, trucks, buses, two-wheeled vehicles, and their authorized service centers, with wheel bearings, tapered roller bearings, small deepgroove ball bearings, seals, special products and spare parts kits (Figure 3).

The Automotive Market is subject to enormous time and cost pressures due to global competition. In order to set itself apart from the competition, SKF invests a considerable amount of money in research and development, resulting in numerous innovations. The increasing complexity of the components that need to be embedded in an overall solution means that the amount of coordination required between SKF and its customers naturally also increases.

Many SKF's products for the Automotive Market are designed based on customer specifications, which is why developers have to work closely with OEMs and tier-1 suppliers. To ensure the greatest possible proximity to customers, application development is decentralized to a great extent (Figure 4). It involves teams comprising of rarely more than 12 members of staff that are distributed over 14 development sites in ten countries. Cross-site collaboration is facilitated by the fact that SKF uses the CAD software Pro/ENGINEER throughout the corporation and manages the CAD data centrally using the PLM solution Windchill PDMLink. As far as external data communication is concerned, however, this means that data constantly needs to be converted into other formats. 


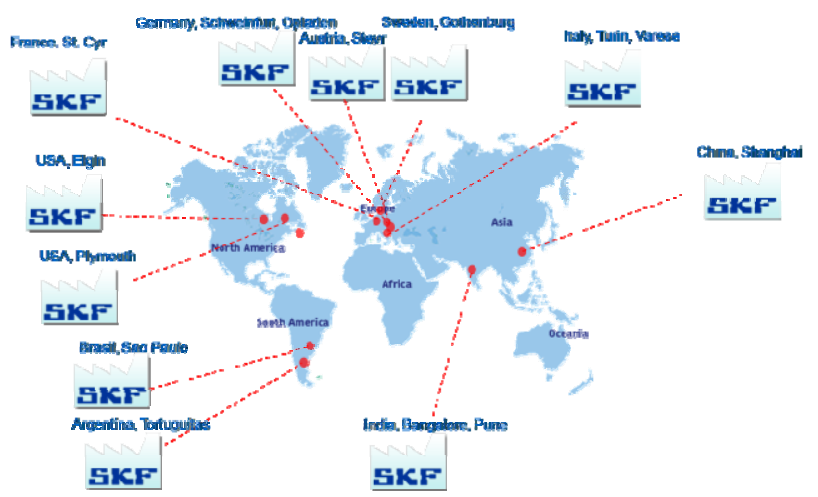

Fig. 4. SKF design centers worldwide

\section{Requirements and Weakness in the Customer Processes}

Where data communication is concerned, automotive suppliers have to take numerous requirements into consideration that are not always easy to fulfil. Not only do they have to make their CAD data available in the format of the authoring systems used by their customers, but they also need to maintain their respective system environments (system configuration, version, starting model, etc.), which sometimes vary from one vehicle program to another. They have to log exchange processes so that they can provide proof of who received which version of the data when. The changes in the development process require a high degree of automation to make the increasing number of exchange processes manageable. At the same time, aspects such as intellectual property protection (IPP) must be given due consideration when converting data since it is possible that the project partners in the distributed development network are competitors. Last but not least almost each OEM prefers the use of a rich PDM client of his own PDM system on the supplier site for the data export and import. Such client must be bought and licensed, and presupposes an expensive internet access with high bandwidth. That is the reason why many suppliers employ additional resident engineers on the corresponding OEM's sites who do nothing else but data management.

Even if the engineers at SKF are developing bearings and seals for tier-1 suppliers, they often have to send the CAD data to the automotive OEM in question because the components involved are safety relevant and have to be simulated in the context of the whole vehicle. As a rule, the OEM's own data exchange specifications are used, which means that suppliers have to make the data available in the format used by the OEM's CAD system. As far as SKF was concerned, this meant that the company had to support approximately 20 different CAD configurations with several versions of a single CAD system. Data exchange was not even straightforward with customers who, like SKF, used Pro/ENGINEER.

The requirements relating to the quality of the data to be exchanged are not uniform throughout the entire development process, something which must be taken into 
consideration when designing the exchange process. As long as the design is being harmonized and optimized in a dialog with engineers at the OEM or tier-1 supplier, requirements relating to data quality are not excessively high. Subsequent to formal release, however, the procurement managers insist on the data being made available according to the contractually agreed guidelines. These guidelines fill volumes since they include not only the data format to be supplied but also the standards, protocols and methods that must be adhered to for the actual data exchange [5]. In many cases a significant manual rework is necessary to supply the data according to those requirements.

A great deal of know-how is embedded in the parametric design models of the bearings as features, formulas and dependencies, and this know-how must not end up in the wrong hands. In the past, the different translation processes meant that a great deal of manual intervention was required to simplify the data during translation and avoid revealing too much intellectual property [6]. The assemblies could not simply be converted into a single part because certain attributes of the individual parts such as, for example, mass or center of gravity, are needed for the calculations and simulations [7]. Certain details do, however, have to be removed as they could reveal how the engineers at SKF solved certain problems from a technical point of view. Thus an additional step became necessary to derive the customer models from the original models.

As is the case with many automotive suppliers, data communication at SKF in the past was characterized by heterogeneous translation and exchange processes, which made traceability more difficult and time-consuming. Translation and data exchange were not integrated, which meant that the files passed through numerous hands. Although the quality of the converted data normally satisfied customer requirements, the costs involved in preparing and transferring the data were relatively high and the data was not always delivered on time. Many weak points were identified.

In addition to Pro/ENGINEER, several business units of SKF Automotive also maintained other CAD systems whose sole purpose was to convert the Pro/ENGINEER data into the respective target format and adapt/rework the converted models and drawings in particular. Maintaining this menagerie of CAD systems alone was costly, without including the time required for user training. And if the specialist for a certain CAD system went on holiday or changed jobs, there was always the danger of not being able to deliver data on time.

Most business units in the Automotive Market had the local engineering service provider, who maintained a close relationship with the respective OEM and was familiar with its CAD requirements, convert the data. However, the collaboration with different partners was not ideal - neither with regard to cost nor in lead time. Bearings do not involve a large volume of data, which meant that the amount of data to be converted for each partner was not very big. As a result, the translation orders never qualified for the more favorable terms and were never processed the fastest.

Another cost driver was data communication itself due to the fact that different, sometimes country-specific, protocols and OEM-specific exchange processes had to 
be supported [8]. SKF normally uses the OFTP protocol in conjunction with ENX, but several customers ask for ENGDAT, which required additional software and support capacity from the outsourced IT department [9]. In addition, customer requirements sometimes change from one project to the next [10]. In view of the large number of parallel projects, it was becoming increasingly difficult to organize the data exchange processes in line with current requirements (Figure 5).

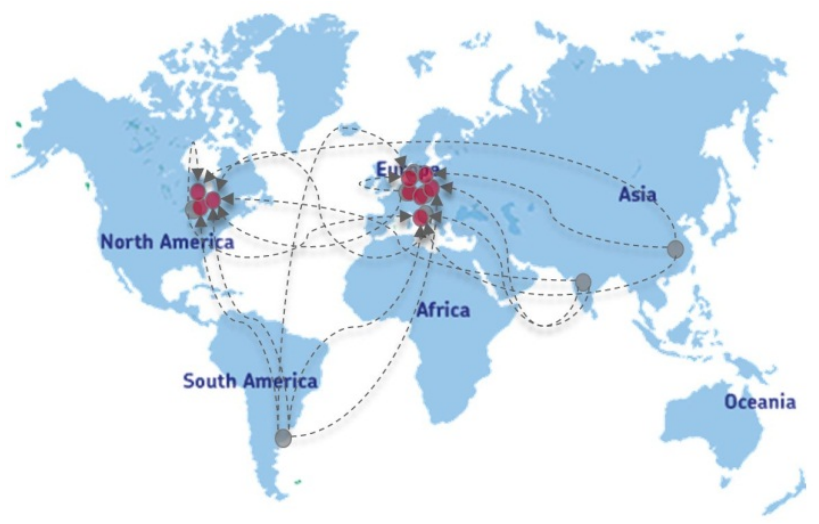

Fig. 5. Data flow required for Global data exchange

\section{Solution Approach}

Since the data communication is not one of the core competences of an automotive supplier, there the question arises as to whether the companies want to take this task into their own hands or whether they should outsource it to an external service provider [11]. The answer depends on the number of exchange partners involved, the variety of the exchange scenarios to be supported, the data exchange frequency and volume of data to be exchanged [12]. In the case of SKF Automotive, outsourcing data translation and exchange promised a return on investment of less than three years. The bearing manufacturer reached an agreement on a cost model that comprises a flat rate for maintaining the services and a rate for the converted and exchanged data volume. This typical pay-per-use scenario makes the costs predicable and accountable for each development project.

To make data communication more efficient, SKF Automotive chooses a uniform solution for all the application areas and sites. A key requirement of the solution and one of the primary reasons for selecting the dedicated service provider was the provision of both data translation services and the organization of data exchange processes with SKF's customers (OEM and tier-1 suppliers) (Figure 6). SKF selected a central service provider for the customer process so that it could avoid having to develop competences of its own if possible. The web-based OpenDESC.com portal offered the added advantage that it required almost no infrastructure nor initial implementation effort and could therefore be rolled out at the various SKF sites very quickly. The basic prerequisites for use of OpenDESC.com are the web browser and fast internet access. 
As technological base the OpenDXM software is used which allows workflow automation of typical data management activities in engineering like import, export, convert, check, send, receive by embedding the corresponding software tools (CAD, PDM, check tools, EDI.). The communication logic of SKF including all communication partners and corresponding persons is stored in the OpenDXM database and controls the data exchange processes preserving a high level of traceability. The software is installed at PROSTEP site including all communication components and devices (ENX, EDI). The access by the end-users at SKF site is given with a web-interface.

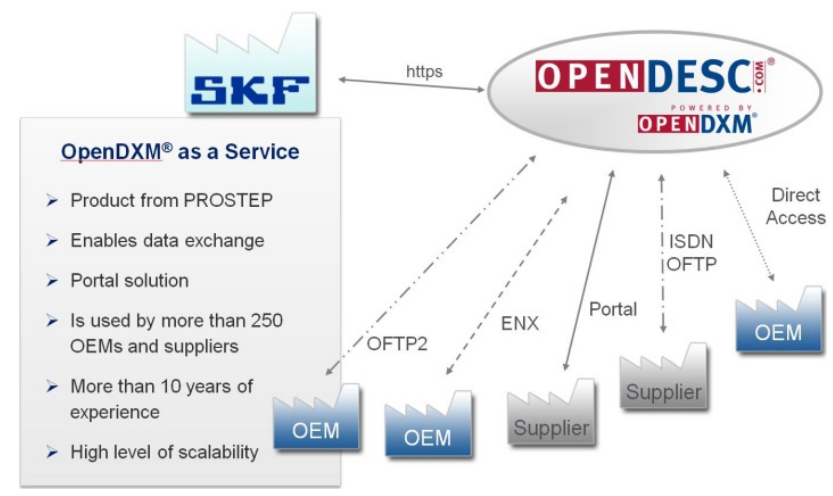

Fig. 6. Data exchange CAD via www.OpenDESC.com

Following a short period of preparation, SKF Automotive started using the central translation and data exchange service at the beginning of 2008. Mapping the exchange partners with their customer-specific requirements regarding translation, quality assurance and data availability took just a few weeks since the data exchange specialists at PROSTEP were able to take advantage of a large pool of existing profiles. Data communication functioned straightaway, with just one exception. There was initially a slight coordination problem with one OEM, which the PROSTEP specialists were able to solve quickly and to the users' complete satisfaction. Since then, the solution has been running smoothly.

To quickly bring users up to a uniform level of learning, key users were named throughout the company. These users were given half a day's training in how to use the solution, and today they are the people which the broad mass of users contact, if they have problems. In addition, PROSTEP offers webinars that allow users to improve their knowledge of the solution and reduce the number of operating errors. In principle, any user at the company today can initiate an exchange process. All they have to do is $\log$ on to www.OpenDESC.com, upload the data and select the exchange partner - all the other processes are executed automatically in the background, or if problems occur, they are solved by the translation specialists at PROSTEP within the framework of the agreed terms of delivery [13] (Figure 7). Despite of high progress of 
the information technology there are still task which must be done manually (repair of grave data quality problems, accurate comparison of drawings). Users at SKF have readily accepted the solution because the amount of time and effort required for data communication has been noticeably reduced.

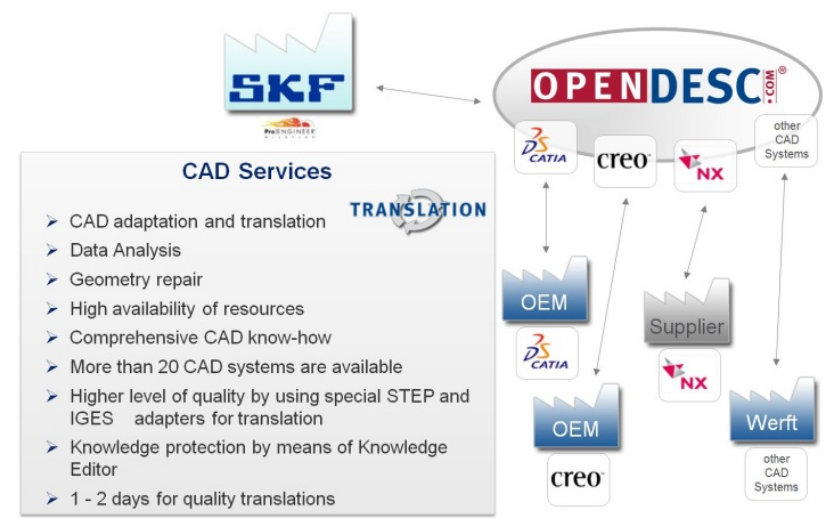

Fig. 7. CAD translation via www.OpenDESC.com

Today, the portal solution is being used at all relevant development sites. A total of more than 100 exchange relationships with the corresponding translation requirements are currently being stored, not all of which, however, are always active. The solution is currently being used for communication with almost 50 OEMs and five tier-1 suppliers at almost 90 locations (Figure 8).

The relatively low number of suppliers can be explained by the fact that SKF uses the normal supplier portal to exchange data with smaller manufacturing partners since the data exchange requirements involved are not as high. The suppliers often also receive drawing data. Last year, SKF converted approximately 4 GB of data via the PROSTEP portal, which was a greater increase than originally planned.

With the reduction of the number of tools for Data Exchange in SKF the company has achieved an ROI of 2 years. This is excluding the increased efficiency by using dedicated trained resources. Therefore the outsourcing is a success for SKF (Figure 8).

The most important benefit of the solution is knowing, that customers will receive the desired data at the agreed point in time and with the stipulated level of quality. Compliance with delivery dates has improved perceptively, which means that customers can be promised delivery on a certain date with a clear conscience. The company expects to reap additional benefits from linking the portal solution to the PLM system Windchill, which will allow data exchange to be better integrated in the customer's engineering change processes. 


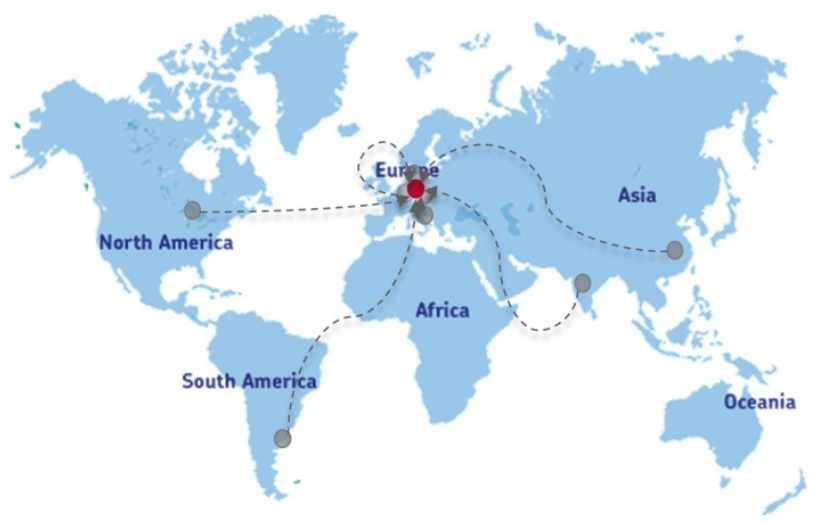

Fig. 8. Data flow when using www.OpenDESC.com

\section{Conclusions and Outlook}

In a dynamic collaborative environment like global automotive industry the working conditions are undertaken continuous change. Suppliers who work together with different OEMs and tier-1 suppliers have to constantly cope with new requirements relating to exchange partners, data formats, system environments to be supported, quality and security requirements, etc. If they take data communication with their customers into their own hands, this means that they have to constantly adapt their data translation and exchange processes to the ever-changing requirements. This involves considerable administrative overhead in terms of time and money, which can on occasion have a negative impact on quality and adherence to deadlines. Collaboration with a competent service provider is therefore an interesting alternative as it not only cuts costs but also facilitates making the exchange processes uniform and ensures a higher level of reliability and traceability.

Good example for the long-term stability of SKF's approach is the recent move of Daimler by replacing CATIA with NX. While the other suppliers which provide the data communication by their own are forced to adapt their infrastructure, processes and methods to new environment at Daimler by spending a significant amount of money and time, SKF has no need for action because this scenario is included in their service contract with OpenDESC.com.

Whether outsourcing is worthwhile depends on various factors such as the number of exchange partners involved, the volume of data, requirements regarding data quality, etc. The example provided by SKF, however, makes it clear that the ROI for such an investment can be calculated relatively well.

The future development belongs the further automation of whole communication process and provision of "communication plugin" [14] for each OEM based on recent standards (STEP AP242 and JT) to avoid the expensive point-to-point connection. 


\section{References}

1. Stark, J.: Global Product. Strategy, Product Lifecycle Management and the Billion Customer Question. Springer, London (2007)

2. Dyer, J.H.: Collaborative advantage: winning through extended enterprise supplier networks. Oxford University Press (2000)

3. Tang, D., Chin, K.-S.: Collaborative Supplier Integration for Product Design and Development. In: Wang, L., Nee, A.Y.C. (eds.) Collaborative Design and Planning for Digital Manufacturing, pp. 99-116. Springer, London (2008)

4. VDA, VDA Empfehlung 4961/3, Abstimmung der Datenlogistik in SE-Projekten, VDA, Frankfurt (2012)

5. Fischer, T., Martin, H.P., Endres, M., Stjepandic, J., Trinkhaus, O.: Anwendungsorientierte Optimierung des neutralen CAD-Datenaustausches mit Schwerpunkt Genauigkeit und Toleranz. VDA, Frankfurt (2000)

6. Liese, H., Rulhoff, S., Stjepandic, J.: Securing Product Know-how by Embedding Intellectual Property Protection into the Organization. In: Proceedings of ICE 2010 Conference (2010)

7. Wendenburg, M.: Kein Datenaustausch ohne Datensicherheit. Digital Engineering, 1 (2008)

8. Hering, U.: Beim Datenaustausch macht Masse die Klasse. In: CADCAM 2008, 3 (2008)

9. Barkholz, D.: Trans-Atlantic networks link up to ease automotive data sharing. Automotive News, 26 (Juli 2010)

10. Wendenburg, M.: Ein System für alle Datenaustauschfälle. In: CADCAM 2006, 7-8 (2006)

11. Sendler, U.: Komplexität ist die größte Herausforderung. In: CADCAM 2007, 9-10 (2007)

12. Wendenburg, M.: Konvertierung als Dienstleistung. In: CADCAM 2007, 9-10 (2007)

13. Reim, R., Cordon, P., Hund, A., Stjepandic, J.: CAD-Konvertierung: Motivation, Probleme, Lösungen. In: CADCAM 2007, 9 (2007)

14. Mervyn, F., Kumar, A.S., Nee, A.Y.C.: A 'Plug-and-Play' Computing Environment for an Extended Enterprise. In: Li, W.D., et al. (eds.) Collaborative Product Design and Manufacturing Methodologies and Applications, pp. 71-91. Springer, London (2006) 